\title{
Dome-Shaped Ellipsoidal Reflector Antenna for UHF-RFID Readers with Confined Near-Field Detection Region
}

\author{
Hsi-Tseng Chou, Hsiang-Ting Cheng, Sheng-Ju Chou, Li-Ruei Kuo, Alice Buffi, and Paolo Nepa
}

\begin{abstract}
This letter proposes and demonstrates the concept of ellipsoidal reflector antennas for radio frequency identification reader applications at UHF band. The antenna can be potentially integrated with environmental structures to confine the reader detection region. The energy bounding characteristics result from the dual-focus feature of an ellipsoidal reflector in its near field region, as the feed located at one of the two foci can create a focused field distribution around the other focus. An axial energy focusing is, thus, formed to confine the energy in a restricted region (near-field beam focusing), also minimizing the interference effects outside of the targeted area. Both numerical simulations and experimental results are presented to demonstrate the feasibility of this antenna concept.
\end{abstract}

Index Terms-Ellipsoidal reflector antenna, near-field (NF) focused antenna (NFFA), NF focused reflector, radio frequency identification (RFID) reader antenna.

\section{INTRODUCTION}

The applications of radio frequency identification (RFID) systems [1] have been significantly extended to the global scope of the Internet of Things, including gate access control, electronic tolling collection, logistics and hospital asset monitoring among others. To assure an accurate detection, many applications need for a restricted area for the tag detection, such as in security identification, financial transactions and warehouse portals. Moreover, the complex environmental structures may result in interferences that cause tag detection failures, namely false negative/positive readings. As a result, the site survey is a very important step when deploying the RFID system, and the surrounding structures could also be used as an opportunistic structure to implement the reader antenna. In this context, near field focused antennas (NFFAs) [2], [3] have been proposed to assure the tag detection in a confined area, with a minimal radiation outside of the area of interest. Since most of NFFAs are electrically large in size [4] to guarantee an effective focusing effect, this paper presents an alternative design concept for RFID reader antennas, which is based on an ellipsoidal reflector structure [5] that can be integrated into environmental structures such as in buildings with a dome-shaped entry area for gate access control. This type of building architecture is quite popular in modern public buildings, as theaters and malls (Fig. 1) that employ dome-shaped structures as a roof. In this case, the relatively low operating frequencies of standardized UHF-RFID systems result in an antenna size that can match quite well to the large physical dimensions of typical dome-shaped roofs. The near-field (NF) focused ellipsoidal reflector could be seamlessly integrated into the dome structure to confine the field distribution in the target area underneath the dome, also minimizing the interferences arising from the scattering by other structures close to the reader antenna. Due to its dual-focus feature, the ellipsoidal reflector may radiate NFs that are axially focused [6] to provide a cylindrical tag detection zone in front of the reflector.

The paper is organized as follows. In Section II, the NF reflector antenna design concept is presented, together with numerical simulations of the field distribution in the NF region. The antenna prototype and system-level experimental results are presented in Section III to demonstrate the validity and feasibility of the antenna design. Finally, concluding remarks are discussed in Section IV.

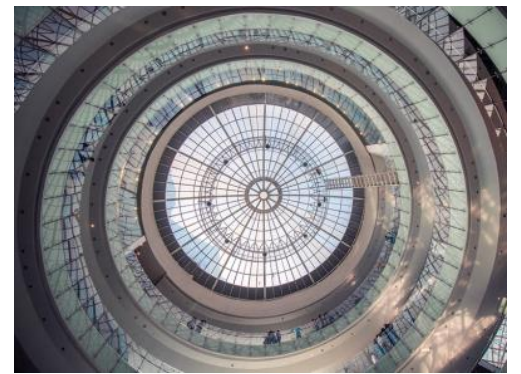

Fig. 1. An example of a large dome-shaped roof where an ellipsoidal reflector antenna for UHF-RFID applications could be integrated. 


\section{ANTENNA CONCEPT AND DESIGN IMPLEMENTATION}

\section{A. Basic Characteristics of the Ellipsoidal Reflector}

The reflector antenna shown in Fig. 2 is formed as a part of a rotationally symmetric ellipsoid described by

$$
\frac{z^{2}}{a^{2}}+\frac{x^{2}+y^{2}}{b^{2}}=1
$$

where $a$ and $b(a>b)$ are the length of the two principal axes, as illustrated in Fig. 2(a). In a practical application, the reflector is implemented with its radiation aperture facing downward to form a vertical cylindrical detection zone. The dual optical foci at $F_{1}$ and $F_{2}\left(z= \pm c\right.$, with $\left.c=\sqrt{a^{2}-b^{2}}\right)$ make all rays launched from $F_{1}$ experience a reflection from the ellipsoid surface, and sum in phase at $F_{2}$, so achieving the NF focusing around $F_{2}$. The designed reflector configuration is shown in Fig. 2(b); $(a, b)=(2,1.5) \mathrm{m}$ and the diameter of the reflector aperture is $2 \mathrm{~m}$. The feed is placed at $F_{1}$, and the distance of the feed from the reflector apex is $a-c=68 \mathrm{~cm}$. The spanned angular range of the feed illumination is $154^{\circ}$.

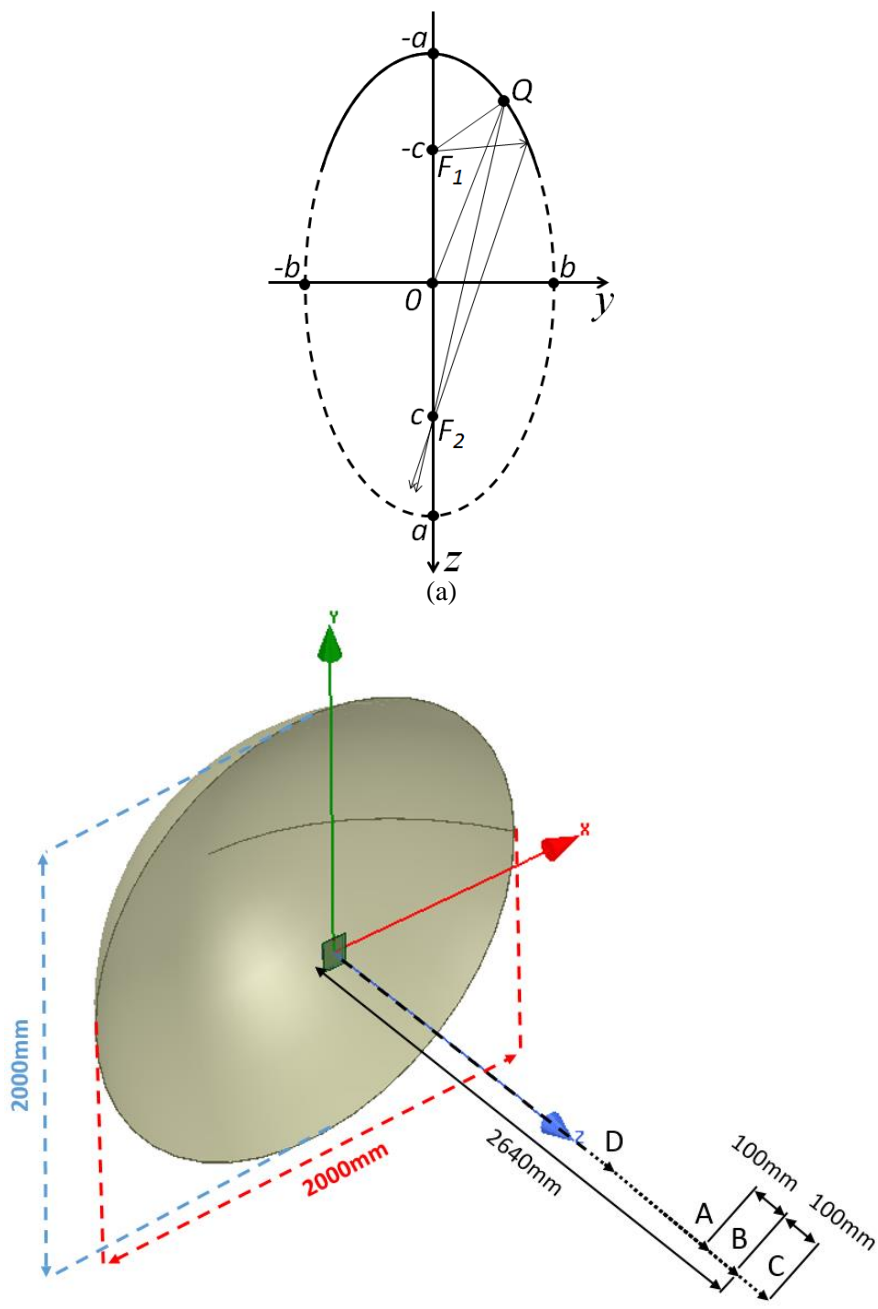

(b)

Fig. 2. Illustration of (a) rotationally symmetric ellipsoid and (b) NF focused reflector antenna that is taken from the top side of the ellipsoid.

\section{B. Feed Antenna Radiation Characteristics}

The feeder is a linearly polarized patch antenna [7], with a 50- $\Omega$ input impedance. The patch antenna is made of two FR4 printed circuit board substrates $\left(\varepsilon_{r}=4.4\right.$, thickness $\left.=1.6 \mathrm{~mm}\right)$ and its geometry is sketched in the inset of Fig. 3. A 9mm-thick air gap is between the two substrates to broaden the impedance frequency bandwidth. The patch size is $l \times w=140.5 \mathrm{~mm} \times 104 \mathrm{~mm}$ and the ground-plane size is $L \times W=157 \mathrm{~mm} \times 120 \mathrm{~mm}$. Then, the resonating length of the radiating patch is roughly $0.44 \lambda$, with $\lambda$ being the wavelength at $915 \mathrm{MHz}$. The length of the feeding coplanar waveguide is $s l=40 \mathrm{~mm}$. The simulated reflection coefficient obtained by using HFSS numerical tool is shown in Fig. 3 (solid line), also accounting for the presence of the reflector. 
The antenna resonates at around $915 \mathrm{MHz}$, with a 40-MHz bandwidth that accommodates for the whole UHF-RFID band in Taiwan: 922-928 MHz, with ERP<1W (ERP-effective radiated power).

It is common using circularly polarized antennas for RFID readers to get a successful reading independently of the tag orientation. On the other hand, here a linearly polarized antenna has been used as the main goal is to show the focused reflector antenna design concept.

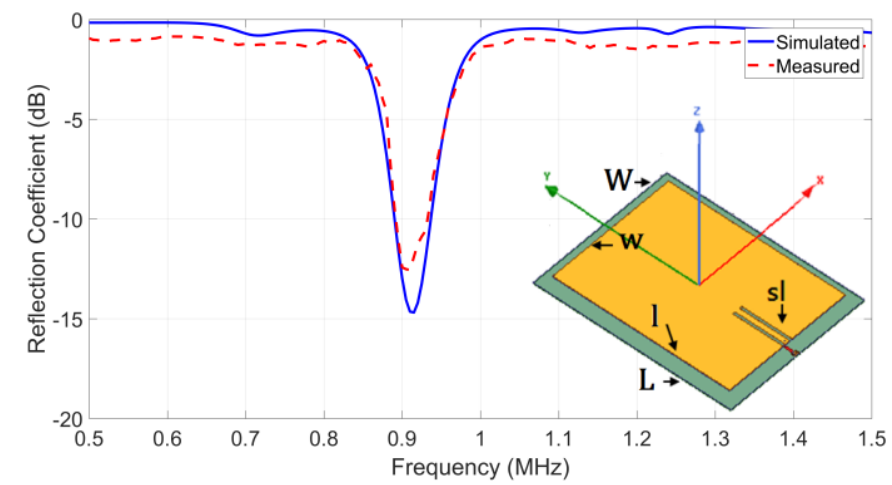

Fig. 3. Measured (dashed line) and simulated (solid line) reflection coefficients at the input port of the reflector feed, which is a linearly polarized patch antenna (shown in the inset).

The feed radiation patterns on the two principal planes, at $925 \mathrm{MHz}$, are shown in Fig. 4. The measured antenna gain and radiation efficiency for the stand-alone feed antenna are $7.4 \mathrm{dBi}$ and $71 \%$, respectively. The cross-polarization level is roughly $-20 \mathrm{~dB}$. The $-\mathrm{dB}$ beamwidths on the two principal planes are roughly $178^{\circ}\left(H\right.$-plane) and $132^{\circ}(E$-plane).

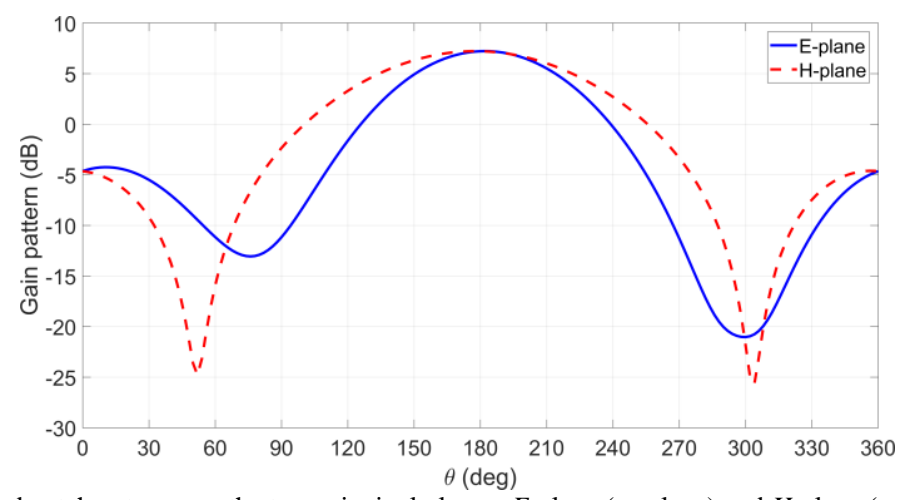

Fig. 4. Measured gain patterns of the feed patch antenna on the two principal planes: $E$-plane ( $y z$-plane) and $H$-plane ( $x z$-plane).

\section{Simulated NF of the Ellipsoidal Reflector}

The contour plot of the NF distributions of the electric field amplitude on the $x z$ - and $x y$ planes are shown in Fig. 5 and Fig. 6, respectively, where the origin $z=0$ has been assumed as coincident with the feed location. The NF distributions are evaluated at $z=254 \mathrm{~cm}$ (A-point), $z=264 \mathrm{~cm}$ (B-point, namely the focal point $F_{2}$ ), $z=274 \mathrm{~cm}$ (C-point) and $z=152 \mathrm{~cm}$ (D-point) where the field peak occurs as apparent from the axial field distribution in Fig. 5.

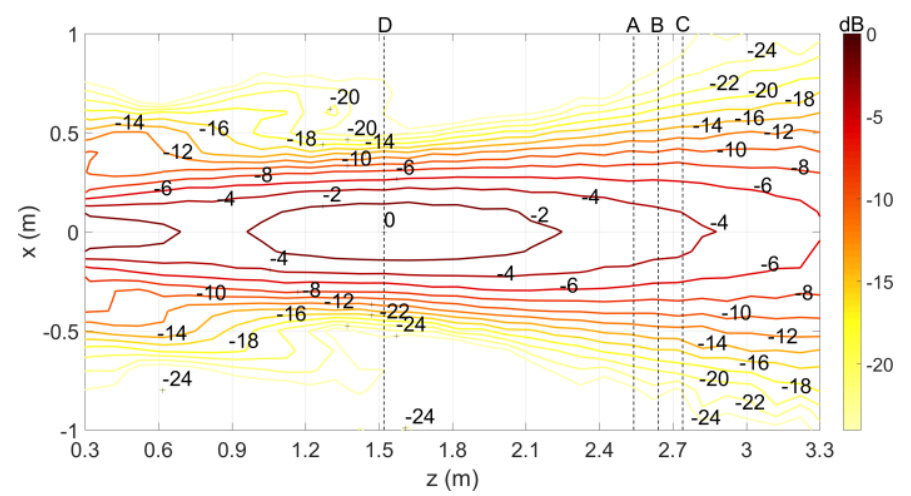

(a) 


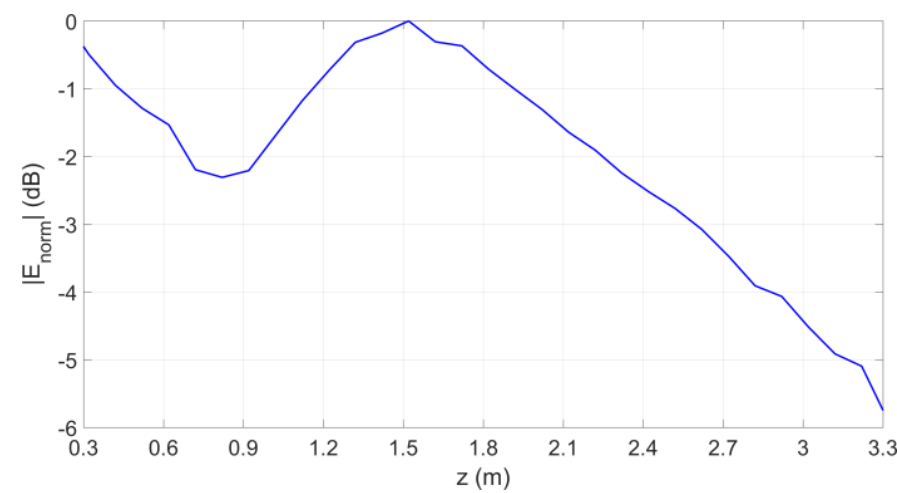

(b)

Fig. 5. NF contour plot (a) on the $x z$-plane and (b) along the reflector axis. In the figures, the reference plane $z=0$ is assumed at the feed location, and the field amplitude is normalized to the value at the field peak position at $z=152 \mathrm{~cm}$.

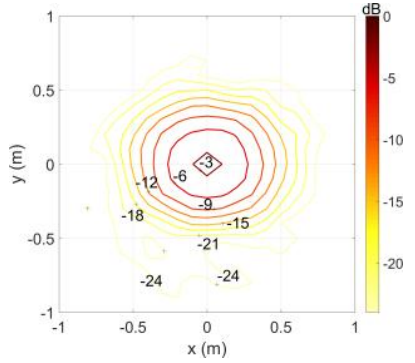

(a)

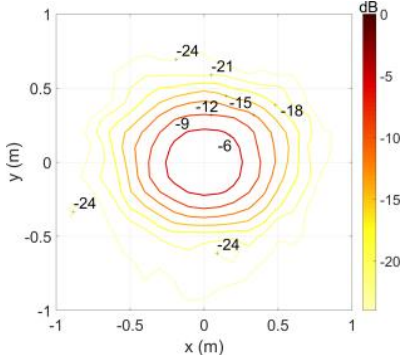

(c)

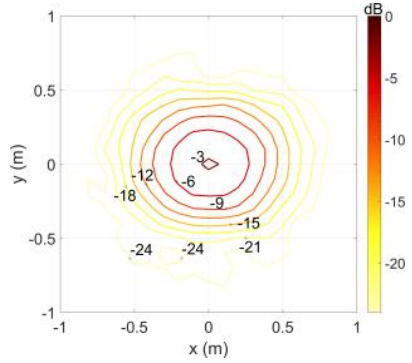

(b)

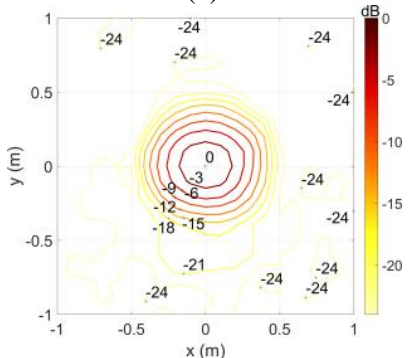

(d)

Fig. 6. Near-field contour plot on $x y$ planes passing through the points (a) $z=254 \mathrm{~cm}$ (A-point), (b) $z=264 \mathrm{~cm}$ (B-point), (c) $z=274 \mathrm{~cm}$ (C-point) and (d) $z=152 \mathrm{~cm}$ (D-point). Note that the reference plane $z=0$ is assumed at the feed location, and the field amplitude is normalized to the value at the field peak position at $z=152 \mathrm{~cm}$.

Indeed, due to the propagation spreading factor [2], [5], the NF distribution along the reflector axis ( $z$-axis) shows a peak value at a distance from the feed location that is smaller than the distance from the feed location of the second focus $F_{2}$. By properly modifying the ellipsoid sizes, it is possible to recover the above focal shift in such a way that the field peak occurs at the assigned point. In any case, it is apparent that the achieved axially focused field distribution can improve tag detection performance in a size-limited region close to the focus at $F_{2}$. Additionally, outside this region, the sharp field drop-off reduces the interferences caused by the scattering that may originate by obstacles close to the reader antenna. Due to the rotational symmetry of the reflector, the pattern on the $y z$ plane does not differ significantly from that on the $x z$ plane, and it is not shown for brevity.

Since a UHF-RFID reader is required to satisfy the ERP regulations in the far-field region, the reflector gain patterns are also investigated. The gain patterns on the two principal planes are shown in Fig. 7. The reflector antenna has a simulated gain of $17.4 \mathrm{dBi}$, with a $3-\mathrm{dB}$ beamwidth of around $24^{\circ}$ on both principal planes. It is worth noting that, in the far-field region, the focused antenna is less directive than a parabolic reflector with the same diameter and feed. Indeed, the parabolic reflector would exhibit an antenna gain greater than $24.7 \mathrm{dBi}$ with a $3-\mathrm{dB}$ beamwidth close to $12^{\circ}$ on both principal planes. This result is in agreement with the well-known defocusing effect that is typical of NFFAs in their far-field region [2]. The latter is an additional advantage of the NFFAs, as they can be used to simultaneously increase the NF amplitude at the focal region and limit the interferences in the antenna far-field region [2]. 


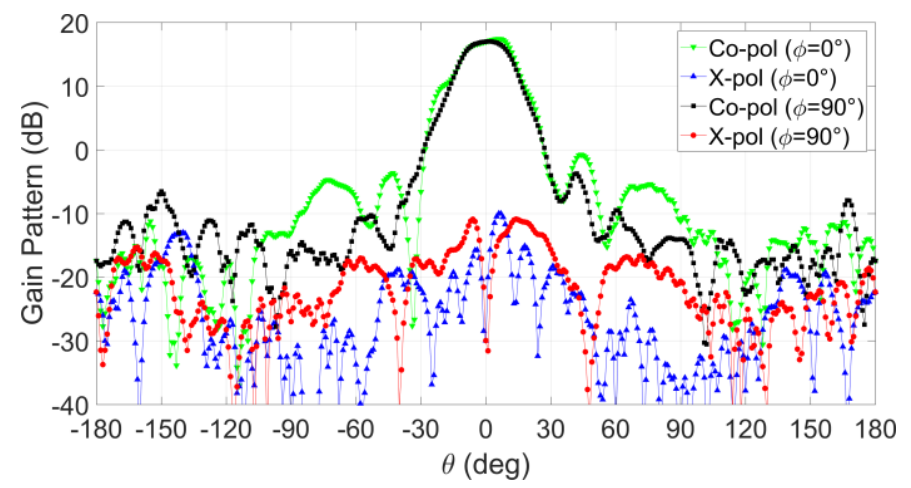

Fig. 7. Simulated co-polar and cross-polar gain patterns of the ellipsoidal reflector, at both principal planes ( $\phi=0: H$-plane; $\phi=\pi / 2: E$-plane).

\section{ANTENNA PROTOTYPING AND EXPERIMENTAL MEASUREMENTS}

The reflector antenna prototype was realized by using meshed steel wires (6 $\mathrm{mm}$ diameter), to form a basic ellipsoidal reflector. Aluminum sheets were afterward employed to cover the external surface. The feed is hung below the reflector by using two wires, and it is connected to a RFID reader through a coaxial cable. The apex of the reflector is $4 \mathrm{~m}$ high from the floor to resemble a typical gate access scenario. Thus, the second focus is at $68 \mathrm{~cm}$ above the floor. Since the fields start defocusing after they penetrate the second focus, the detection zone is reasonably defined in the range beyond $60 \mathrm{~cm}$ from the floor. The antenna prototype is shown in Fig. 8. The measured reflection coefficient (shown in Fig. 3, with a dashed line) is in good agreement with simulated results.

The RFID measurement setup is also shown in Fig. 8. The Astra UHF reader by ThingMagic and the HLN7014A tag by Motorola have been used during the tests (both support the EPC Class $1 \mathrm{G} 2$ protocol). By considering that the simulated reflector far-field gain is equal to $17.4 \mathrm{dBi}$, the reader output power has been set at $10 \mathrm{dBm}$ to be compliant with the ERP limit (ERP $<30 \mathrm{dBm}$ ).

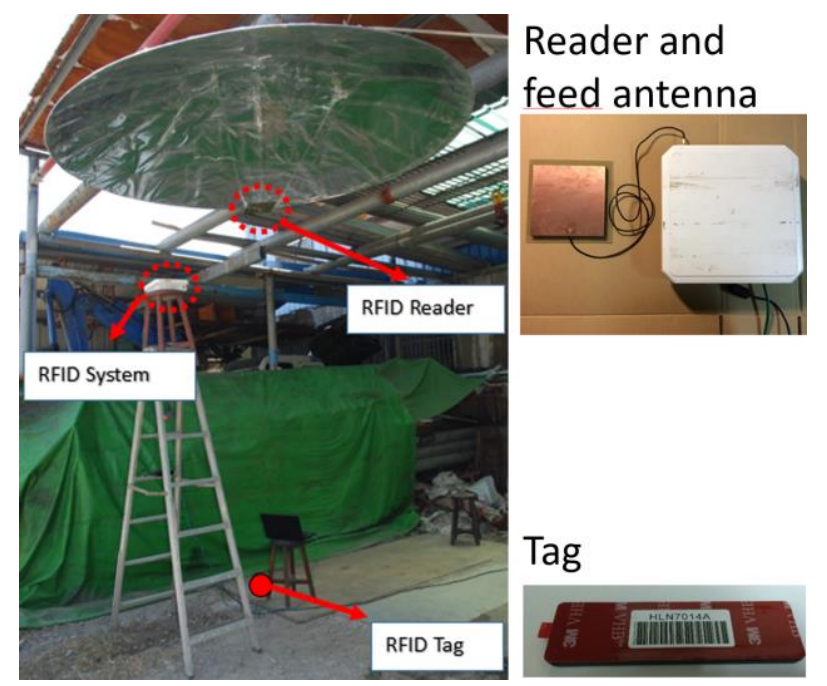

Fig. 8. Measurement setup with the RFID system equipped with the ellipsoidal reflector reader antenna.

The RFID system performance has been verified in terms of tag detection capability on the planes passing through the points at A, B and C of Fig. 2. The tag has been moved on a $2 \times 2-\mathrm{m}^{2}$ grid, with a step of $10 \mathrm{~cm}$. The results of the readability tests are shown in Fig. 9 , where only the locations of successful detection are marked. It appears that the reading zone is limited to an area with a size of around 1-1.2 m. Only minor variations can be observed between the results for the three planes, thus confirming the similarities between the simulated contour plots in Fig. 6 .

\section{CONCLUSION}

An ellipsoidal reflector antenna suitable to radiate an axially focused NF has been proposed for RFID applications at the UHF band. The antenna can potentially be integrated into a dome-shape environmental structure. Numerical and experimental results have successfully demonstrated its NF focusing radiation characteristics. The antenna implementation scenario can be extended to treat a variety of similar environmental architectures, such for example the sun-shielding umbrellas in outdoor café shops. 


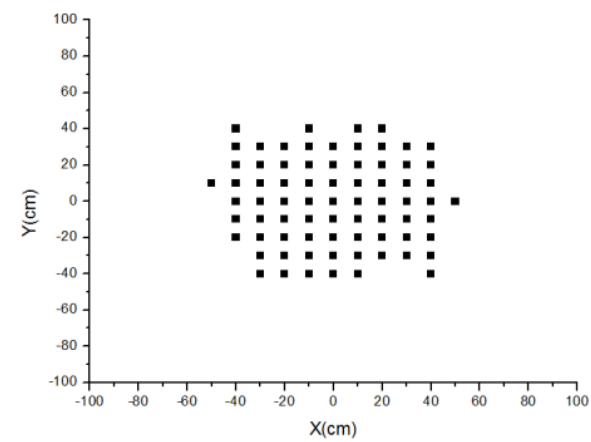

(a)

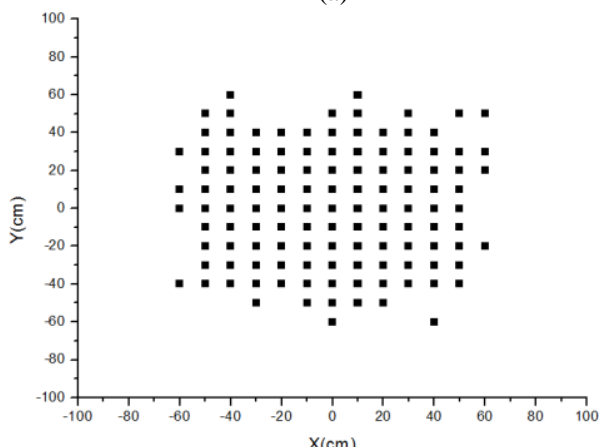

(b)

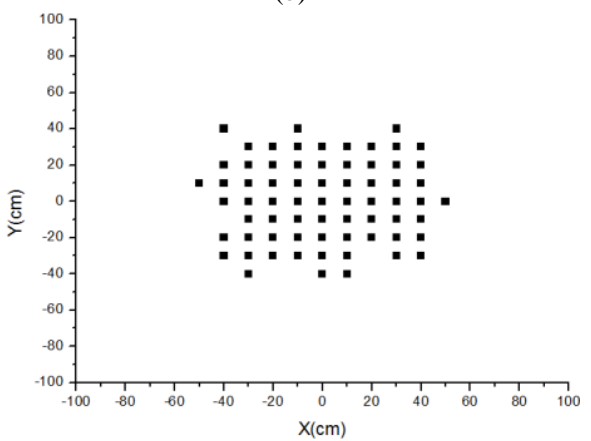

(c)

Fig. 9. RFID reader detection area when the NF focused ellipsoidal reflector antenna is fed with a $10 \mathrm{dBm}$ input power, on three different planes: (a) $z=254 \mathrm{~cm}$ (A-point); (b) $z=264 \mathrm{~cm}$ (B-point); (c) $z=274 \mathrm{~cm}$ (C-point).

\section{REFERENCES}

[1] R. Gadh, P. Chu, G. Q. Huang, K. Michael, B. S. Prabhu, G. Roussos, "RFID - A Unique Radio Innovation for the 21th Century," Proceedings of the IEEE, vol. 98,(9), pp. 1546-1549, Sept. 2010.

[2] P. Nepa and A. Buffi, "Near-field-focused microwave antennas: near-field shaping and implementation," IEEE Antennas and Propagation Magazine, vol. 59(3), pp. 42-53, 2017.

[3] P. Nepa, A. Buffi, A. Michel, and G. Manara, "Technologies for Near-Field Focused Microwave Antennas," International Journal of Antennas and Propagation, vol. 2017, ID 7694281, 17 pages, 2017.

[4] H.-T.Chou, T.-M.Hung, N.-N.Wang, H.-H.Chou, and P.Nepa, "Characteristic comparisons between array, reflectarray and elliptic reflector antennas in RFID near-field focused communications," IEEE International Conference on Wireless Information Technology and Systems (ICWITS '10), pp. 1-4, Honolulu, Hawaii, USA, 2010.

[5] H- T. Chou, L. R. Kuo, H.-H. Chou, K. L. Hung and P. Nepa, "Realistic implementation of ellipsoidal reflector antennas to produce near-field focused patterns," Radio Science, Volume 46(5), pp. 1-10, October 2011.

[6] Z. Li, Z.Wang, J.Wu, and H. Tian, "Axially steered spots of near-field Fresnel zone plate lens with sweeping frequency," IEEE Antennas and Wireless Propagation Letters, vol. 13, pp. 955-958, 2014.

[7] R. Garg et al., Microstrip Antenna Design Handbook, Artech House, 2001. 